\title{
Capture PCR: Efficient Amplification of DNA Fragments Adjacent to a Known Sequence in Human and YAC DNA
}

\author{
Maria Lagerström, Jüri Parik, Helena Malmgren, \\ Jason Stewart, Ulf Pettersson, and UIf Landegren
}

Department of Medical Genetics, Uppsala Biomedical Center, Uppsala University, S-751 23 Uppsala, Sweden

We have devised a procedure, termed capture PCR (CPCR), that permits the rapid isolation of DNA segments situated adjacent to a characterized nucleotide sequence. In this procedure, a DNA sample is restriction-digested and a linker, comprising two base-paired oligonucleotides, is added to the ends by ligation. Multiple extension reactions are performed using a biotinylated primer derived from the known sequence, permitting the subsequent isolation of extension products on a streptavidin-coated support. The enriched fragments are amplified exponentially using another specific oligonucleotide, hybridizing $3^{\prime}$ to the biotinylated primer in combination with one of the linker oligonucleotides, now functioning as a PCR primer. The convenience of CPCR is greatly enhanced by using a novel streptavidin-coated manifold, which is constructed so that it projects into each individual well of a microtiter plate. The procedure permits the simultaneous isolation of fragments from large numbers of DNA samples and minimizes the risk of contamination between reactions. We have applied this method to identify DNA sequences located downstream of known sequences in the human genome. The technique has also been used to identify end fragments of sequences cloned in a yeast artificial chromosome (YAC) vector. The reactions can be initiated directly from yeast colonies and provide access to DNA sequence information for these end fragments in a minimal number of steps. With the aid of the present technique, we have isolated over 100 end fragments from YACs derived from the human $X$ chromosome. Isolated end sequences have been used to order YAC clones into a contig.

$\mathbf{T}$ he PCR technique provides for the highly specific amplification of unique DNA segments defined by two surrounding primer sequences. (1) A number of procedures have been developed to apply PCR in circumstances where sequence information is available from only one side of a segment of interest. For example, in inverse PCR, DNA fragments are ligated into circles. ${ }^{(2-4)}$ Thus, a known DNA sequence can be made to surround sequences normally located on both sides of this DNA segment, permitting their amplification using defined primer sequences. A series of other methods, sometimes termed anchor $\mathrm{PCR},{ }^{(5-9)}$ avoid the requirement for circularization by the addition of oligomers to all ends in a fragmented DNA preparation. These oligomers may be added by enzymatic tailing, ${ }^{(5,6)}$ or by ligation, ${ }^{(7)}$ and can then be used as complements for a general amplification primer. This primer is used in conjunction with a specific primer to amplify an interven- ing DNA segment. The oligomers that are added to all termini may be designed so that a complement for the general primer is formed only after a molecule has been copied from a specific primer hybridizing internal to the fragment. This is done to avoid amplification of all DNA sequences surrounded by oligomers. For instance, in the vectorette strategy, or bubble $\mathrm{PCR},{ }^{(8,9)}$ a duplex oligonucleotide with an internal mismatched segment must be copied before a complement for the general primer is formed. Still, in this technique, unlike conventional PCR, priming by a single oligonucleotide is sufficient to generate a template that can subsequentially undergo exponential amplification, greatly increasing the potential for false reaction products.

In contrast, techniques relying on the hybridization of two specific primers afford much greater specificity. ${ }^{(10-14)}$ Mueller and Wold described the use of a specific primer for a first extension reaction in fragmented DNA to generate a blunt end that is then ligated to a blunt oligonucleotide linker. ${ }^{(10)}$ In a subsequent reaction, this fragment is amplified using a linker-primer and a specific primer, located downstream of the one used in the first extension reaction. This technique has proven sufficiently specific to permit the sequencing of singlecopy gene sequences directly from genomic samples. ${ }^{(11)}$

The number of different techniques for amplifying DNA segments bordered on one side only by known sequences 
attests to the importance of the problem as well as to the lack of a clearly superior technique. Existing methods exhibit poor specificity or are cumbersome to perform. We now present a simple and highly specific method to isolate sequences located adjacent to a characterized DNA segment based on the hybridization of two specific primers. In this method, a biotinylated, specific primer is used for a first set of extension reactions in DNA modified by the addition of oligonucleotide linkers to all restriction ends. Extension products, enriched for the sequences of interest, are captured on a novel streptavidin-coated support. The relevant fragments are then amplified with a primer representing the oligonucleotide linker and a primer hybridizing downstream of the biotinylated oligonucleotide. This principle of physically enriching extension products using a biotin-streptavidin interaction for subsequent amplification has been developed independently by Rosenthal and Jones. ${ }^{(14)}$ We demonstrate in this paper that the technique is of sufficient specificity to identify unique sequences in the human genome. The streptavidin-coated sup- port permits the simultaneous processing of large numbers of reactions and greatly reduces the potential for contamination between samples. Furthermore, we show that, with a standard set of oligonucleotides, end fragments from YAC vector inserts may be isolated and sequenced using a rapid protocol starting directly from yeast colonies.

\section{METHODS \\ Reagents}

Oligonucleotides were synthesized by standard phosphoramidite chemistry. The sequences of the oligonucleotides employed are given in Table 1. A 5' biotin residue was incorporated by reacting a hydroxysuccinimide ester of biotin (Clonetech) with an oligonucleotide having a primary amino group added as an amino-linker residue. The reaction products were purified by FPLC over a reverse-phase $\mathrm{C} 18$ column. Alternatively, a biotinylated phosphoramidite residue was directly added during oligonucleotide synthesis (Biotin $\mathrm{dX}$, Midland Certified Reagents Co, TX).

\section{Capture PCR}

Genomic DNA was digested with restriction enzymes and, in the same reaction, linkers were ligated to the DNA fragments (Fig. 1). To avoid contamination, tips with barriers (Aerosol Resistant Tips, Continental Laboratory Products) were used for pipetting throughout the protocol. All the reactions were performed in flexible microtiter plates (Falcon 3911). For the combined restriction and ligation reaction, $100 \mathrm{ng}$ of DNA was incubated with a pair of oligonucleotide linkers ${ }^{(10)}$ (Table 1) at $2 \mu \mathrm{M}$ each, one end of which can join to restriction ends without recreating the recognition sequence. One-half microliter of restriction enzyme (usually approximately 5 units, depending on the concentration) and $0.5 \mu \mathrm{l}$ of T4 DNA ligase (4 units, Pharmacia) were added to a final volume of $10 \mu \mathrm{l}$ containing $0.5 \mathrm{~mm}$ ATP, 10 mmoles Tris- $\mathrm{HCl}$ ( $\mathrm{pH} \mathrm{7.5),} 10$ mmoles $\mathrm{MgCl}_{2}$, and 50 mmoles potassium acetate. The reaction was incubated at $37^{\circ} \mathrm{C}$ for $3 \mathrm{hr}$.

When intact yeast cells were used as a source of DNA, a single colony was resuspended in $50 \mu \mathrm{l}$ of $\mathrm{H}_{2} \mathrm{O}$. Five microliters of the suspension was added

TABLE 1 Sequence of Oligonucleotides Used in the Present Work

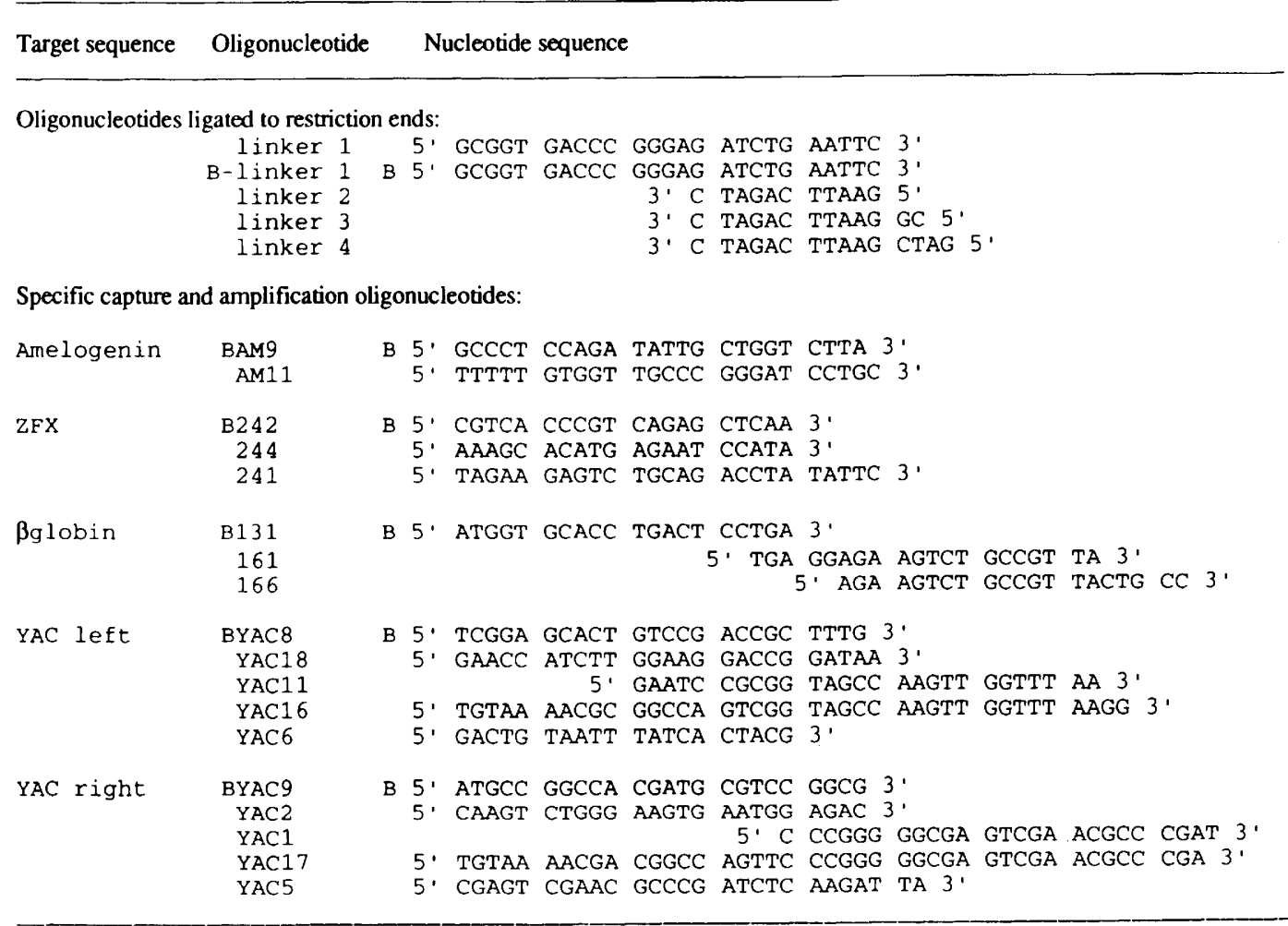




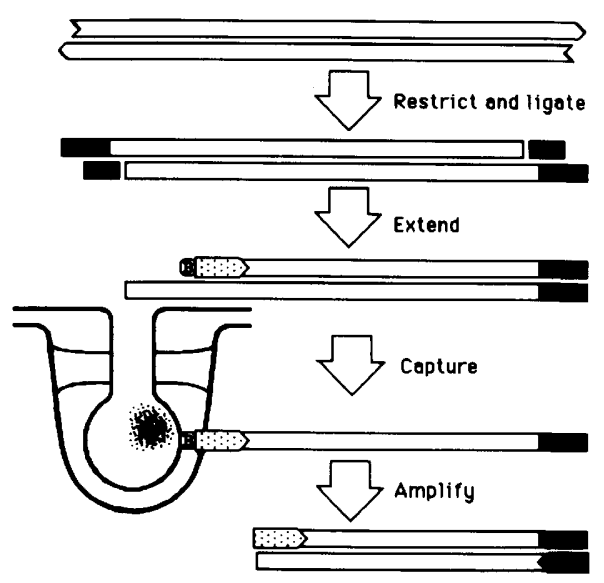

FICURE 1 A schematic description of capture PCR and of the solid support used. Linker oligonucleotides are shown in black, and primers specific for a given genomic region are dotted. Genomic DNA is digested with a restriction enzyme, and, in the same reaction, a linker is ligated to the cleavage site. The DNA sample is subjected to extension reactions under PCR conditions, using a $5^{\prime}$ biotinylated primer. The reaction products extend from the primer across the DNA fragment of interest and terminate in a sequence complementary to the added linker. Biotin-labeled extension products are captured on a 96-pronged streptavidincoated mainfold projecting into individual microtiter wells. After washes, a PCR is performed using as templates the isolated extension products bound to the support. A second specific primer, hybridizing downstream of the biotinylated oligonucleotide, is used as a PCR primer together with the linker oligonucleotide. The PCR yields a product containing the DNA segment of interest.

to a final volume of $10 \mu \mathrm{l}$ containing $0.1 \mu \mathrm{M}$ biotinylated YAC vector primer in a standard $P C R$ reaction mix of 50 $\mathrm{mm}$ Tris- $\mathrm{HCl}$ (pH 8.3 at room temperature), $50 \mathrm{~mm} \mathrm{KCl}, 1.5 \mathrm{~mm} \mathrm{MgCl}_{2}, 200$ $\mu \mathrm{M}$ each of dATP, dCTP, dGTP, and dTTP, $12.5 \mu \mathrm{g}$ bovine serum albumin $/ \mathrm{ml}$, and 0.035 units $/ \mu$ l of AmpliTaq (Cetus). The sample was overlayered with mineral oil and denatured at $93^{\circ} \mathrm{C}$ for $5 \mathrm{~min}$, followed by annealing at $55^{\circ} \mathrm{C}$ for $1 \mathrm{~min}$, and extension at $72^{\circ} \mathrm{C}$ for $3 \mathrm{~min}$ in two cycles before proceeding with the restriction-ligation step as described above, but in a final volume of $20 \mu \mathrm{l}$.

Multiple rounds of extension reactions were performed from a biotinylated primer. One-half microliter of the restriction-ligation reaction was added to a $20-\mu 1$ standard PCR reaction containing $0.1 \mu \mathrm{M}$ biotinylated primer. The temperature was varied between $94^{\circ} \mathrm{C}$ for $1 \mathrm{~min}, 55^{\circ} \mathrm{C}$ for $1 \mathrm{~min}$, and $72^{\circ} \mathrm{C}$ for $2.5 \mathrm{~min}$ in 35 cycles followed by a final incubation at $72^{\circ} \mathrm{C}$ for 7 $\min$.

The biotin-labeled extension prodducts were immobilized on a commercially available polystyrene device, configured as a microtiter plate lid with eight rows of 12 pin-and-ball extensions projecting into individual microtiter wells (Falcon 3931). A number of means of covalently coating the 96 prongs of the support with streptavidin were examined and will be presented elsewhere (J. Parik et al. in prep.). A preliminary protocol, resulting in a binding capacity of around 1 pmole of biotinylated product per prong, is available upon request. Before use, the support was briefly incubated in a washing solution of $0.1 \mathrm{M}$ Tris- $\mathrm{HCl}(\mathrm{pH}$ 7.5), $1 \mathrm{M} \mathrm{NaCl}$, and $0.1 \%$ Triton $X-100$, and then incubated for $3 \mathrm{hr}$ at room temperature in the wells of the extension reactions. As an alternative, $40 \mu \mathrm{l}$ of prewashed streptavidin-coated magnetic particles (Dynal A.S., Norway) were added to the extension reaction and incubated at room temperature. The solid supports were subsequently washed in individual microtiter wells once with washing solution, once with a denaturing solution of $0.01 \mathrm{M} \mathrm{NaOH}$, $1 \mathrm{M} \mathrm{NaCl}, 0.1 \%$ Triton $\mathrm{X}-100$, and again with washing solution. Finally, the solid supports were washed in PCR buffer.

The immobilized extension products were used as templates in a PCR reaction. The streptavidin-coated device was present during the entire PCR. If streptavidin-coated paramagnetic beads were used, $2.5 \mu \mathrm{l}$ of immobilized extension products, resuspended in $10 \mu \mathrm{l}$ of PCR buffer, were employed as template. The 50- $\mu$ l PCR reactions contained the oligonucleotide linker 1 and a specific primer (Table 1), representing a sequence located downstream of the biotinylated primer, at $1 \mu \mathrm{M}$ each. The temperature was varied as described above.

The identity of amplified DNA sequences was confirmed by three different methods: (1) A new PCR was performed using a specific primer located downstream of the previous $\mathrm{PCR}$ primer (Table 1). One-half microliter of product from the original PCR was used as template in a 50- $\mu$ l PCR with 1 $\mu \mathrm{M}$ each of the internal primer and linker 1 (Table 1). The reaction products were analyzed by agarose gel electrophoresis to identify fragments exhibiting the expected change in size upon using the internal primer. (2) Samples from the original PCR were separated in an 1.5\% agarose gel and appropriate fragments excised from the gel and diluted in $200 \mu \mathrm{l}$ of $\mathrm{H}_{2} \mathrm{O}$. After melting at $95^{\circ} \mathrm{C}, 1 \mu \mathrm{l}$ of the samples was used as templates in a 50- $\mu \mathrm{l}$ PCR, using the specific primer together with a primer representing sequences located downstream of the specific primer in the opposite orientation. (3) The CPCR fragments were digested with restriction enzymes. Twenty-five microliters of the reaction product from the original PCR was incubated with 40 units of a restriction enzyme, known to cut downstream of the specific primer, in a 3 -hr incubation at $37^{\circ} \mathrm{C}$. The digested samples were separated in a $1.5 \%$ agarose gel.

\section{Southern Blot Analysis of YACs}

DNA segments isolated by CPCR from ends of inserts in YACs were labeled by random priming (Oligolabelling kit, Pharmacia). The labeled ends were prehybridized with fragments from the YAC vector, amplified using the oligonucleotides YAC2 and YAC18 (Table 1), to ensure that vector-derived portions of the probes did not contribute to the hybridization signal. The probes were hybridized to blots prepared from genomic DNA from a set of YAC clones and the yeast wildtype strain AB1380, digested with HindIII, and separated by agarose gel electrophoresis. ${ }^{(15)}$

\section{Sequence Analysis of Fragments Generated by CPCR}

The nucleotide sequence of CPCR products was obtained by solid-phase sequencing ${ }^{(16)}$ and analyzed by automated fluorescent sequencing. Onehalf microliter of the product of the original PCR was amplified in a $50 \mu \mathrm{l}$ PCR with $0.1 \mu \mathrm{M}$ linker 1 , modified by the addition of a $5^{\prime}$ biotin group, and $1 \mu \mathrm{M}$ of a specific primer with the M13 forward primer sequence incorporated in the $5^{\prime}$ end (see Table 1). The PCR 
was performed for 30 cycles. Twenty microliters of the reaction was incubated with $20 \mu \mathrm{l}$ of prewashed streptavidin-coated paramagnetic beads for $30 \mathrm{~min}$ at room temperature. The bound PCR products were resuspended in $100 \mu \mathrm{l}$ of $0.1 \mathrm{~N} \mathrm{NaOH}$, $1 \mathrm{M} \mathrm{NaCl}$ for $15 \mathrm{~min}$ at room temperature, followed by three washes in the same solution and one in $10 \mathrm{~mm}$ Tris$\mathrm{HCl}(\mathrm{pH} \mathrm{7.5)}$. The final wash was performed with $100 \mu \mathrm{l} 80 \mathrm{~mm}$ Tris- $\mathrm{HCl}$ (pH 8.9), $20 \mathrm{~mm}$ ammonium sulfate, and $5 \mathrm{mM} \mathrm{MgCl}_{2}$ and the samples were resuspended in $6 \mu \mathrm{l}$ of this buffer. The sequencing reactions were performed in 30 temperature cycles with fluorescent labeled M13 forward primers, using the Taq Dye Primer Cycle Sequencing Core Kit (Applied Biosystems) according to the manufacturer's protocol. The four PCR reactions with differently dye-labeled M13 primers and each of the four different dideoxynucleotides were pooled and precipitated. The dried pellet was resuspended in deionized formamide and $50 \mathrm{~mm}$ EDTA, 5:1 (vol/vol), and loaded on an automated DNA Sequencer (Applied Biosystems, Model 373A).

\section{RESULTS}

\section{Isolation of Human DNA Sequences} by CPCR

PCR achieves its high specificity as a consequence of the requirement that two oligonucleotide primers must hybridize under relatively stringent conditions in the appropriate orientation near each other for an exponential amplification to result. (1) We examined the possibility of obtaining the same specificity by physically isolating extension products from one specific primer and subsequently using these enriched fragments as templates for PCR by applying another, downstream primer in conjunction with a general primer.

First, stocks of modified genomic DNA were prepared by ligating linkers, made up of two base-paired oligonucleotides, to ends generated by restriction digestion (Table 1 ). The oligonucleotide linkers lack 5' phosphate groups, ensuring that only one of the oligonucleotides is covalently joined to the ends of the genomic DNA fragments. The 25-mer oligonucleotide linker $1^{(10)}$ can be used together with one of three different short complementary oligonucleotides to modify ends generated by more than 30 different restriction enzymes. The short oligonucleotides have low GC content, ensuring that they will not function as primers in PCR reactions. The oligonucleotide dimers are designed so that the ligation of dimers to restriction ends will not regenerate the recognition sequence of the restriction enzymes. Therefore, restriction digestion and ligation can be performed simultaneously. This ensures the gradual accumulation of the desired restriction fragments terminated by a standard 25-mer sequence. The linker-modified DNA samples can be saved and stored for later use with any set of two specific CPCR primers.

Aliquots of the linker-modified DNA samples were mixed with a specific, biotinylated primer and subjected to 35 cycles of extension reactions, interfoliated with denaturation and annealing steps. Biotinylated extension products were captured on a 96-pronged, disposable support projecting into individual microtiter wells. Streptavidin, covalently coupled to the solid support, efficiently immobilized the biotinylated molecules. The device permits the simultaneous washing and transfer of sets of 96 reaction intermediates between microtiter plates. Furthermore, it greatly reduces the risk of contamination between reactions. Commercially available streptavidincoated paramagnetic particles may also be used to capture biotinylated extension products, but the handling of large numbers of samples is less convenient and increased care has to be taken to avoid contamination between reactions.

After washes, extension products bound to the supports were transferred to another set of microtiter wells. They were used as templates in a PCR reaction containing a primer hybridizing downstream of the biotinylated one and another primer, representing the linker sequence added to restriction ends in the genomic DNA sample (Fig. 1). The amplification products were inspected after agarose gel electrophoresis. Figure 2 demonstrates an array of fragments, defined on one side by a primer sequence and on the other by the presence of a restriction site.
The primers were derived from the human amelogenin, (17) ZFX, (18) and $\beta$ globin genes. ${ }^{19)}$ The amelogenin reactions are shown directly after CPCR, whereas $\mathrm{ZFX}$ and $\beta$-globin fragments were first subjected to gel purification and then analyzed as detailed below.

The identity of the isolated amelogenin fragments was confirmed by reference to a restriction map. ${ }^{(17)} \mathrm{CPCR}$ products of the correct size were obtained for each of the four investigated restriction enzymes that cut within 2 $\mathrm{kb}$ downstream of the CPCR primers (Fig. 2 and data not shown). The $\beta$ globin- and ZFX-related fragments were excised from an agarose gel, diluted, and subjected to another PCR reaction. For fragments amplified using primers derived from the ZFX gene, the downstream CPCR primer was applied together with a primer located internally to the fragments and in the opposite orientation. All four identified fragments were shown to contain internal sequences hybridizing to this new primer, establishing that the fragments are all derived from the $\mathrm{ZFX}$ gene or from a closely homolo-gous sequence. For $\beta$-globin-related fragments, a primer located somewhat downstream of the second $\mathrm{CPCR}$ primer was applied in conjunction with the linker oligonucleotide. As can be seen in Figure 2, the fragment obtained from the $\beta$-globin gene after $A l u I$ digestion and one of the Dral fragments underwent the expected slight change of size upon amplification with a PCR primer located internally. The other DraI fragment failed to give rise to the expected amplification product and was scored as an incorrect amplification product. An $A l u$ I site was observed at the appropriate location in the restriction map for the $\beta$-globin gene region. In contrast, no Dral site was seen. However, the $\beta$-globin primer sequences are quite well conserved in the $\delta$-globin gene and a Dral site is present at the appropriate location, downstream of the $\delta$-globin gene. Therefore, it is likely that the observed Dral fragment is derived from the $\delta$-globin gene.

Thus, CPCR is equivalent to conventional $P C R$ in specificity and it may be performed using simple steps and under standardized conditions. As illustrated here, the technique may be applied to restriction map a segment of 


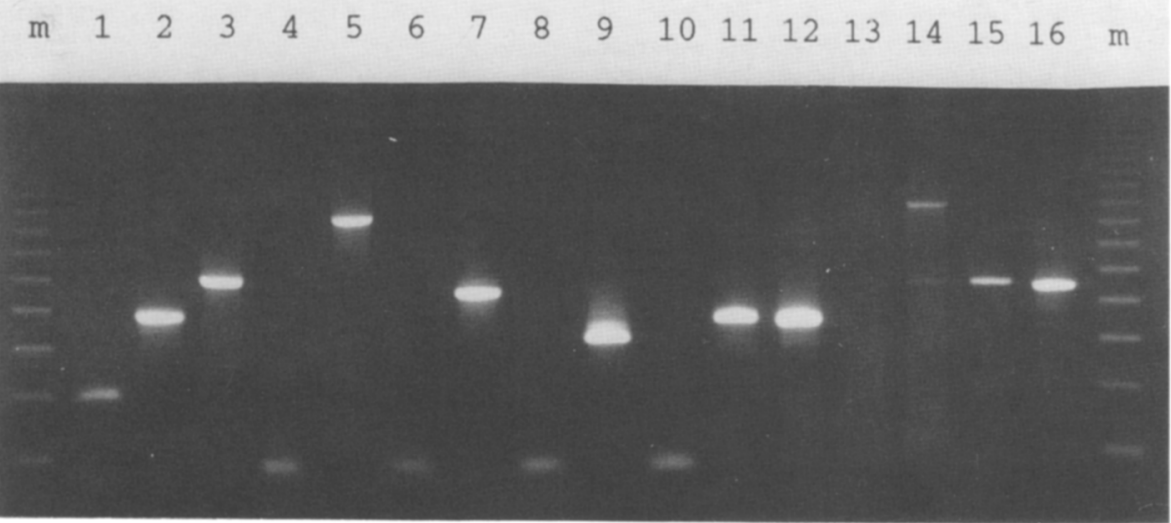

FIGURE 2 Human DNA fragments amplified by CPCR using pairs of primers located in tandem. (Lanes 1 and 2) Amelogenin gene sequences amplified after digestion of genomic DNA with $A l u$ l and Dral, respectively. (Lanes 3-10) Fragments obtained using primers derived from the ZFX gene after digestion with Dral, HincII, Nael, and Pvull (lanes 3, 5, 7, and 9, respectively). The identity of the observed fragments was investigated in the adjacent lanes 4 , 6,8 , and 10 by determining if the excised bands could serve as templates, generating an approximately $100-b p$ amplification product, in a PCR with primers located internal to authentic products. (Lanes 11-16) CPCR products generated using primers derived from the $\beta$-globin gene. A gel-purified product generated after digestion with AluI was reamplified using the same primers (lane 11) or using a specific primer located 5 nucleotides downstream of the previous one (lane 12). Similarily, two products observed after Dral digestion followed by CPCR were reamplified using the same primers (lanes 13 and 15) or with the downstream one (lanes 14 and 16, respectively). The lanes are flanked by a 123-bp ladder (BRL) serving as size markers (lane $m$ ). genomic DNA and to isolate fragments of interest.

\section{YAC End Fragment Isolation by CPCR}

CPCR is well suited for applications where large numbers of DNA samples are searched for DNA segments located adjacent to a standard sequence. One example is the identification of fragments located immediately internal to vector sequences in YACs. YAC vectors represent valuable cloning vehicles that can accept more than a megabase of genomic DNA. Identification of end fragments from YAC inserts provides for rapid genomic walks by strides of several hundred kilobases. ${ }^{(20)}$

End sequences may be obtained from the inserts of all YAC clones using the same standard set of CPCR primers (Table 1). To ensure that the procedure does not isolate sequences present in the normal yeast genome, the biotinylated primers, hybridizing on either side of the YAC vector cloning site, were derived from pBR322 sequences in the vector. Figure 3 demonstrates agarose gel-resolved end fragments from a set of YAC clones. Fre-

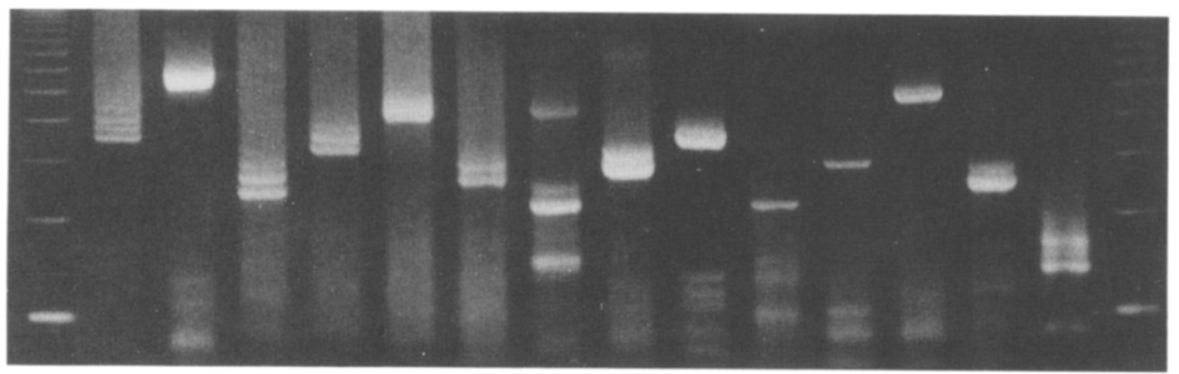

FIGURE 3 Products of CPCR exemplifying the routine isolation YAC end fragments from a series of YAC clones. (Lane 1) CPCR from a left YAC end using the restriction enzyme $B c l$; (lanes 2-14) CPCRs from right YAC ends and using the enzymes BclI, BglII, BglII, HinPI, HinPI, BstUI, DraI, DraI, EcoRV, EcoRV, Hincll, PvuII, and PvuII, respectively. quently, more than a single amplification product is seen upon agarose gel electrophoresis. This could be a consequence of partial digestion during the restriction-ligation step of CPCR or it could result from illegitimate priming events generating shorter end fragments or completely irrelevant amplification products. Authentic fragments are easily identified using an internally located, vector-derived primer. Figure 4 demonstrates the identification of CPCR products as true end fragments by observing the expected difference in size between agarose-separated CPCR products, amplified with amplification primers located at different distances from the cloning site. Moreover, different restriction enzymes generate end fragments of different lengths. Shorter fragments define restriction sites that should be located internally to the longer fragments if these are derived from the same sequence. As illustrated in Figure 4, the presence of such restriction sites may be confirmed by restriction analysis of longer fragments.

\section{Rapid Preparation of Yeast DNA Samples for CPCR}

In CPCR large numbers of samples may be handled in parallel and processed in rapid steps. The purification of DNA from individual YAC clones thus be-
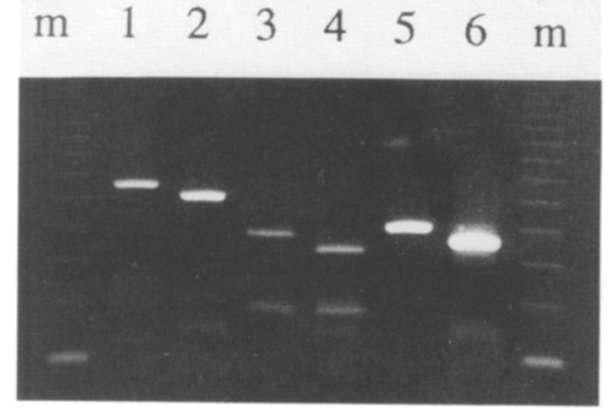

FIGURE 4 Confirmation of the identity of amplification products obtained from YAC clone XY316 using CPCR primers derived from the right-hand side of the vector. The nonbiotinylated vector-specific primer used for the samples separated in lanes 1,3, and 5 hybridizes $90 \mathrm{bp}$ upstream of that used in lanes 2, 4, and 6. Lanes 1 and 2 reveal CPCR products obtained after digestion with AluI. Lanes 3 and 4 represent Rsal digestions of the samples in lanes 1 and 2, respectively. The CPCR reaction products in lanes 5 and 6 were generated after $R s a$ I digestion. 
comes a rate-limiting step. Therefore, we investigated the possibility of performing the isolation of YAC end fragments directly from individual yeast colonies after minimal preparation. Yeast cells were directly added to microtiter wells, overlayered with mineral oil, and heated to $94^{\circ} \mathrm{C}$ for $5 \mathrm{~min}$ followed by primed synthesis of a new strand from a vector-derived primer using Taq polymerase and nucleotides. The primer used was identical to that later required in the extension reactions from a biotinylated primer. The incubation at $94^{\circ} \mathrm{C}$ serves to make the yeast DNA accessible for the subsequent enzymatic reactions while inhibiting nucleases and proteases. The extension reaction renders the DNA segment $3^{\prime}$ to the primer double stranded, as is required for the subsequent restriction digestion and ligation. Figure 5 illustrates that direct end-isolation from yeast colonies generates results similar to those obtained with purified DNA, significantly reducing the turnaround time in a YAC walking procedure.

\section{Hybridization Analysis of the Relative Orientation of a Set of YAC Clones}

Isolated YAC end fragments may be used to identify YAC clones with partially overlapping inserts by applying the fragments as hybridization probes. We used end fragments from three YAC clones to orient these with respect to one another. The three clones were isolated from a YAC library on the

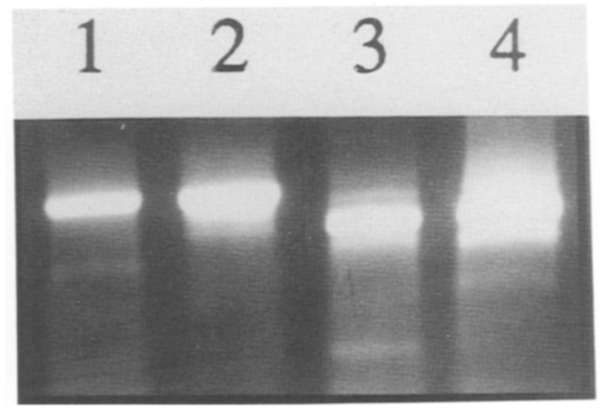

FIGURE 5 Comparison between YAC end isolation by CPCR starting from yeast colonies (lanes 1 and 3 ) or from purified DNA (lanes 2 and 4). Lanes 1 and 2 represent the left end fragment from YAC clone XY492 and lanes 3 and 4 represent the right end fragment from YAC clone XY669. basis of sharing a marker DXS304. This marker is located in the vicinity of the fragile $X$ locus at the distal end of the long arm of the $X$ chromosome. ${ }^{(21)}$ End fragments, isolated by CPCR, were used to probe DNA blots prepared from the relevant yeast clones and wild-type yeast as shown in Figure 6A. As expected, overlapping YAC inserts have at least one end hybridizing to the other in a reciprocal manner. The size of the fragments detected on blots differs between the clone from which the fragment was isolated and overlapping clones because the end fragments are interrupted by vector sequences. The hybridization pattern allows deduction of the order and orientation of the YACs with respect to one another as indicated in Figure 6B. Moreover, the analysis establishes the order of the isolated end fragments along the chromosome, providing a physical map of this region. This orientation of the YAC clones has been established independently (M. d'Urso and D. Schlessinger, pers. comm.).

\section{Sequence Analysis of YAC End Fragments}

Isolated YAC end fragments represent a convenient source of sequence tagged sites, ${ }^{(22)}$ appropriately spaced in the genome. Furthermore, the availability of nucleotide sequence information for the end fragments permits PCR-assisted screening of YAC libraries to identify overlapping YAC clones. ${ }^{(23)}$ We have applied a solid-phase fluorescent sequencing protocol to permit the rapid acquisition of sequence information for newly isolated end fragments. CPCR products of interest were diluted and subjected to one more amplification reaction using a $5^{\prime}$ biotinylated version of linker 1 and a YAC vectorspecific primer including the M13 forward sequencing primer as a $5^{\prime}$ addition (see Table 1). The amplified products were immobilized on streptavidin-coated magnetic particles, denatured and washed, and subjected to multiple rounds of Taq polymeraseassisted extension reactions in the presence of fluorescent-labeled M13 forward sequencing primers. Reaction products were loaded onto an automated DNA sequencer. Figure 7 illustrates sequence data from the end of a 272-bp CPCR product. The YAC end fragment ends with the dinucleotide $A G$, representing a half-site of the restriction enzyme Alul used to generate the fragment, immediately followed by the complement of the linker 1 sequence. The method used is generally applicable for the sequence analysis of fragments isolated by CPCR.

\section{DISCUSSION}

In this paper we describe an efficient technique for the PCR-assisted isolation of DNA segments located adjacent to a known nucleotide sequence. The procedure employs two specific primers, oriented in the same direction, applied sequentially but under standard PCR conditions, i.e., brief annealing and extension at high temperature. Extension products from the first primer are captured on a support before proceeding to an exponential amplification reaction using a downstream specific primer in conjunction with a general primer. The specificity of the technique is similar to conventional PCR permitting isolation of human single-copy sequences. Fragments of up to $2 \mathrm{~kb}$ have been isolated and only small amounts of purified DNA are required in the procedure. Indeed, denaturation followed by an extension reaction is sufficient to render the relevant DNA segment accessible for analysis in a crude sample of cells.

Central to the present strategy is the concept of enriching for molecules extended from a biotinylated primer. Biotinylated extension products may be captured using commercially available streptavidin-coated paramagnetic particles. ${ }^{(14)}$ We have, however, experienced great difficulties handling more than just a few DNA samples at a time using paramagnetic particles as a solid support. Contamination between reactions becomes increasingly difficult to avoid when more than just a few reactions are performed in parallel. In contrast, the solid support used here permits rapid washes and transfer of sets of up to 96 reaction intermediates between different enzymatic milieus with little potential for contamination. This is of particular value in applications involving PCR, where purity rather than large binding capacity is of the greatest importance. It is likely that a capture step will be of general value 


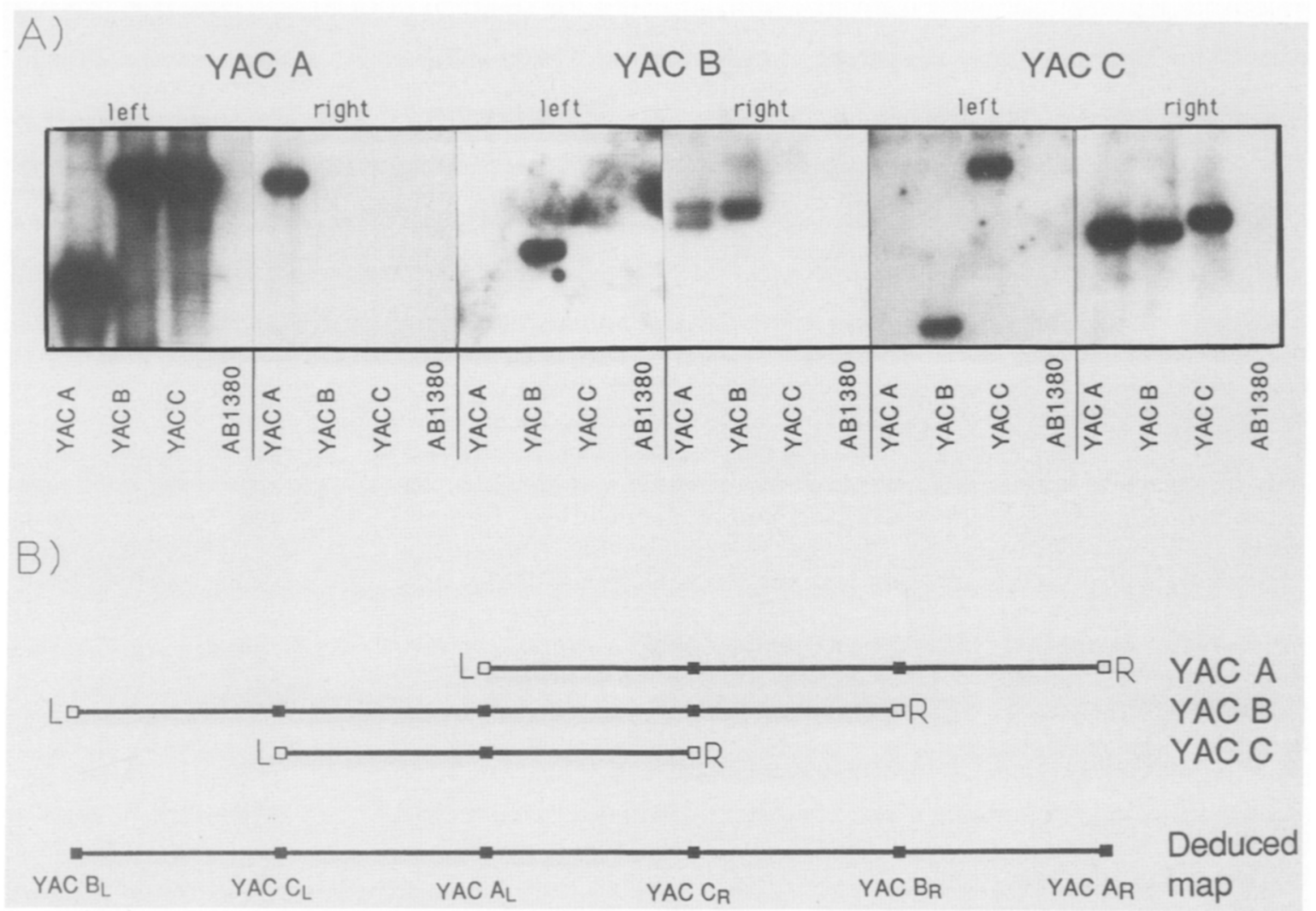

FIGURE 6 Ordering of YAC clones by hybridization of isolated end fragments to Southern blots prepared from HindIII-digested DNA from the same yeast clones and from $\mathrm{AB} 1380$, a yeast strain not harboring any $\mathrm{YAC}$. (A) The results of the hybridization analysis. $(B)$ The relative map order of the YAC end fragments inferred from the hybridization analysis.

in situations where currently nested PCR is used to obtain increased specificity. The capture of specific target sequences by hybridization to a biotinylated primer may also serve as a means to enrich rapidly for relevant sequences to permit specific enzymatic reactions starting from a crude tissue sample. The possibility of handling large numbers of samples using the streptavidin-coated solid phase has proven valuable in several other preparative and analytical molecular genetic techniques and it is particularily helpful in diagnostic applications (M. Samiotakis, unpubl.).

The use of CPCR to isolate end fragments from DNA segments cloned into YAC vectors illustrates an important application of the technique. Here, a standard set of oligonucleotides may be used to process any number of DNA samples. Since the same set of primers is used for all yeast clones, the reaction conditions may be completely standardized. We have used CPCR to isolate a total of more than $100 \mathrm{YAC}$ end fragments. Using the two blunt-endgenerating enzymes, $A l u \mathrm{I}$ and RsaI, YAC end fragments were successfully isolated from both ends of approximately $85 \%$ of the YAC clones investigated. With an extended set of restric-

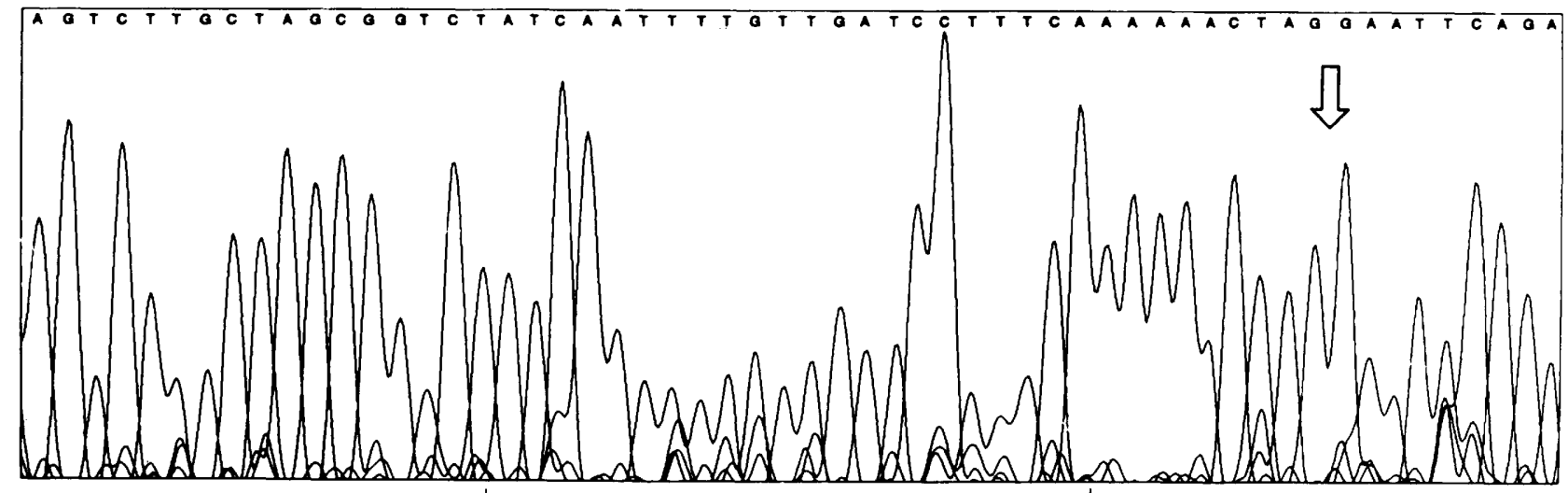

FICURE 7 Results from a solid-phase, fluorescent DNA sequence analysis of a YAC end fragment isolated by CPCR from a YAC clone, digested with Alul. The illustration represents 57 bases of the nucleotide sequence toward the end of the CPCR product. The arrow denotes the beginning of the complement of the sequence of linker 1. 
tion enzymes, no YAC clone has proven recalcitrant to this procedure, although the presence of repeated sequences may limit the usefulness of a given end fragment in genomic mapping.

The speed with which a YAC walking project may be conducted using CPCR is enhanced by the fact that multiple YAC clones may be studied in parallel and the reaction may be inititated directly from a YAC clone seeded on a petri dish without DNA purification.

Alternatively, the reaction may also be initiated from cells scraped from a frozen glycerol stock of yeast cells (data not shown). Therefore, it is possible to obtain end fragments from a large group of identified YAC clones in 1 or 2 working days. The actual hands-on time per set of 96 reactions is only a few hours. Moreover, isolated end fragments may be rapidly processed for DNA sequence analysis. By applying a solid-phase sequencing procedure, no cloning is required and nucleotide sequence information can be acquired for the end fragments within $48 \mathrm{hr}$ of the identification of a given YAC clone. The use of fluorescent-labeled primers representing sequences located $3^{\prime}$ of the previously used YAC-derived oligonucleotides should provide an extra level of specificity and accordingly should further enhance the performance of the procedure.

The CPCR protocol should be of general utility in a number of situations. For example, the isolation of a Dral-limited fragment from the $\delta$ globin gene, using primers derived from the $\beta$-globin gene, illustrates the possibility of applying CPCR to isolate homologous gene sequences. CPCR primers corresponding to a genomic sequence of less than 40 conserved nucleotide positions are sufficient for this purpose. The identity of the obtained fragments may be confirmed using a downstream primer or by sequencing isolated fragments. Other possible applications of CPCR include the isolation of a full-length CDNA sequence from a population of cDNA molecules using primers derived from a short known sequence, from phylogenetically conserved residues, or based on peptide sequence information. Similarily, promoter sequences, intron-exon boundaries, or breakpoints of deletions and translocations may be identified using CPCR. By a simple modification of the standard protocol, CPCR reactions could be performed using randomly fragmented DNA samples, modified with the appropriate linker sequence. Amplification products in a desired size range could then be selected after size separation. The CPCR strategy should also be useful for the isolation of populations of DNA segments located adjacent to a sequence present in multiple sequence contexts in a DNA sample. An example of this would be the definition of multiple viral integration sites in a sample of genomic DNA. Thus, the CPCR procedure represents a generally useful way of applying PCR in contexts where sequences outside of a known DNA segment are sought.

\section{ACKNOWLEDGMENTS}

The competent assistance of Mrs. Elsy Johnson is gratefully acknowledged. Drs. David Schlessinger and Michele d'Urso generously provided YAC clones and information on their orientation. The sequence information for ZFX was kindly supplied by Dr. David Page. Ulf Gyllensten and MarieLouise Steen offered valuable comments on the manuscript. This work was supported by the Beijer foundation, the Swedish National Board for Technical Development, and the Medical Research Council. U.L. is a recipient of a fellowship from the Wallenberg Foundation.

\section{REFERENCES}

1. Saiki, R.K., D.H. Gelfand, S. Stoffel, S.J. Sharf, R. Higuchi, G.T. Horn, and H.A. Erlich. 1985. Enzymatic amplification of $\beta$-globin genomic sequences and restriction site analysis for diagnosis of sickle cell anemia. Science 230: 1350-1354.

2. Triglia, T., M.G. Peterson, and D.J. Kemp. 1988. A procedure for in vitro amplification of DNA segments that lie outside the boundaries of known sequence. Nucleic Acids Res 16: 8186.

3. Ochman, H., A.S. Gerber, and D.L. Hartl. 1988. Genetic applications of an inverse polymerase chain reaction. Genetics 120: 621-623.

4. Silver, J. and V. Keerikatte. 1989. Novel use of polymerase chain reaction to amplify cellular DNA adjacent to an integrated provirus. $J$. Virol. 63: 1924-1928.

5. Loh, E.Y., J.F. Elliot, S. Cwerla, L.L. Lanier, and M.M. Davis. 1989. Polymerase chain reaction with single sided specificity: Analysis of the T cell receptor delta chain. Science 243: 217-220.

6. Frohman, M.A., M.K. Dush, and G.R. Martin. 1988. Rapid production of full length cDNAs from rare transcripts using single gene specific oligonucleotide primer. Proc. Natl. Acad. Sci. 85: 8998-9002.

7. Roux, K.H. and P. Dhanarajan. 1990. A strategy for single site PCR amplification of dsDNA: Priming digested cloned or genomic DNA from an anchor-modified restriction site and a short internal sequence. Biotechniques 8: 48-57.

8. Riley, J.H., R. Butler, D.J. Ogilvie, R. Finniear, D. Jenner, R. Anand, J.C. Smith, and A. Markham. 1990. A novel, rapid method for isolation of terminal sequences from yeast artificial chromsome (YAC) clones. Nucleic Acids Res. 18: 2887-2890.

9. Copley, C.G., C. Boot, K. Bundell, and W.L. McPheat. 1991. Unknown sequence amplification: Application to in vitro genome walking in Chlamydia trachomatis L2. Bioteclmology 9: 74-79.

10. Mueller, P. and B. Wold. 1989. In vivo footprinting of a muscle specific enhancer by ligation mediated PCR. Science 246: 780-786.

11. Pfeifer, G.P., S.D. Steigerwald, P.R. Mueller, B. Wold, and A.D. Riggs. 1989. Genomic sequencing and methylation analysis by ligation mediated PCR. Science 246: 810-813.

12. Fors, L., R. Saavedra, and L. Hood. 1990. Cloning of the shark $P_{0}$ promoter using a genomic walking technique based on the polymerase chain reaction. Nucleic Acids Res. 18: 2793-2799.

13. Parker, J.D., P.S. Rabinovitch, and G.C. Burmer. 1991. Targeted gene walking polymerase chain reaction. Nucleic Acids Res. 19: 3055-3060.

14. Rosenthal, A. and D.S. Jones. 1990. Genomic walking and sequencing by oligo-casette mediated polymerase chain reaction. Nucleic Acids Res. 18: 3095-3096.

15. Southern, E.M. 1975. Detection of 
specific sequences among DNA fragments separated by gel electrophoresis. J. Mol. Biol. 98: 503-517.

16. Hultman, T., S. Stahl, M. Hornes, and M. Uhlén. 1989. Direct solid phase sequencing of genomic and plasmid DNA using magnetic beads as solid support. Nucleic Acids Res. 17: 4937-4946.

17. Nakahori, Y., O. Takenaka, and Y. Nakagome. 1991. A human X-Y homologous region encodes "Amelogenin." Genomics 9: 264-269.

18. Page, D., R. Mosher, E. Simpson, E. Fisher, G. Mardon, J. Pollack, B. McGillivray, A. de la Chapelle, and G. Brown. 1987. The sex-determining region of the human $\mathrm{Y}$ chromosome encodes a zinc finger protein. Cell 51: 1091-1104.

19. Landegren, U., R. Kaiser, J. Sanders, and L. Hood. 1988. A ligase-mediated gene detection technique. Science 241: 1077-1080.

20. Burke, D., G. Carle, and M. Olson. 1987. Cloning of large segments of exogenous DNA into yeast by means of artificial chromosome vectors. Science 236: 806-812.

21. Dahl, N., K. Hammarström-Heeroma, P. Goonewardena, C. Wadelius, K.-H. Gustavson, G. Holmgren, G.J.B. van Ommen, and U. Petterson. 1989. Isolation of DNA probe of potential use for diagnosis of the fragile $X$ syndrome. Hum. Genet. 82: 216-218.

22. Olson, M., L. Hood, C. Cantor, and D. Botstein. 1989. A common language for physical mapping of the human genome. Science 245: 1434-1435.

23. Green, E.D. and M.V. Olson. 1990. Systematic screening of yeast artificial-chromosome libraries by use of the polymerase chain reaction. Proc. Natl. Acad. Sci. 87: 1213-1217.

Received July 22, 1991; accepted in revised form August 15, 1991. 


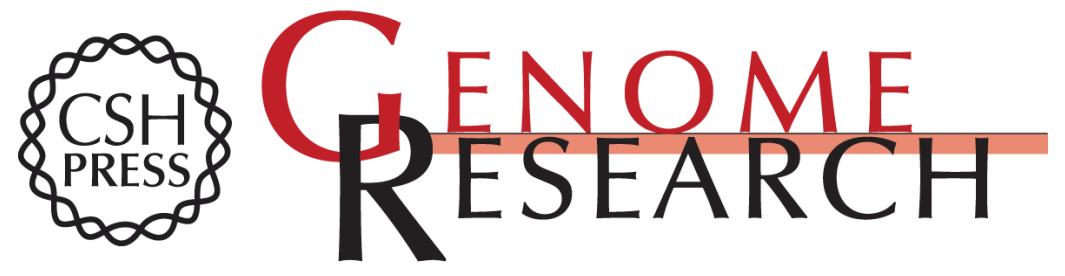

\section{Capture PCR: efficient amplification of DNA fragments adjacent to a known sequence in human and YAC DNA.}

M Lagerström, J Parik, H Malmgren, et al.

Genome Res. 1991 1: 111-119

Access the most recent version at doi:10.1101/gr.1.2.111

References This article cites 23 articles, 11 of which can be accessed free at:

http://genome.cshlp.org/content/1/2/111.full.html\#ref-list-1

\section{License}

Email Alerting Receive free email alerts when new articles cite this article - sign up in the box at the Service top right corner of the article or click here.

\section{Affordable, Accurate Sequencing.}

To subscribe to Genome Research go to:

https://genome.cshlp.org/subscriptions 